(RESEARCH ARTICLE)

\title{
People living with HIV and oral health professionals: What relationship?
}

\author{
Mbathio Diop 1, ${ }^{*}$, Mamadou Diatta ${ }^{2}$, Aida Kanouté ${ }^{1}$, Serigne Dame Dieng 1, Mansoum Ndiaye ${ }^{3}$, Denis \\ Bourgeois ${ }^{4}$, Cheikh MM Lo ${ }^{1}$ and Daouda Faye ${ }^{1}$
}

\begin{abstract}
${ }^{1}$ Department of Odontology, Public Health Office, Faculty of Medicine, Pharmacy and Odonto-Stomatology, Cheikh Anta Diop University of Dakar, Senegal.

${ }^{2}$ Department of Odontology, Dental Surgery Office, Faculty of Medicine, Pharmacy and Odonto-Stomatology, Cheikh Anta Diop University of Dakar, Senegal.

${ }^{3}$ Department of MBA, Health Care, the African Center for Graduate Studies in Management Health Economics of Dakar, Senegal.

${ }^{4}$ Department of Public Health, Faculty of Medicine, Claude Bernard University of Lyon 1, France.
\end{abstract}

Publication history: Received on 13 July 2020; revised on 02 August 2020; accepted on 09 August 2020

Article DOI: https://doi.org/10.30574/wjarr.2020.7.2.0258

\begin{abstract}
The implementation of highly active antiretroviral therapy [HAART] has contributed to the reduction of HIV infectionrelated morbidity and mortality. However, despite efforts in clinical care, most HIV-infected people worldwide will continue to develop oral diseases. Besides, people living with HIV [PLHIV] have unmet needs for oral health care. In this context, the literature on the relationship between dental surgeons and PLHIV was reviewed. The current epidemiology reveals the relevance of promoting oral health to efficiently improve PLHIV's quality of life. Thus, dental services, therefore, can play a role in the primary prevention of the HIV epidemic, through early diagnosis and treatment of oral diseases. This will be based on good hygiene in dental offices, treatment of oral lesions and nutritional and psychosocial support for PLHIV. Containing the pandemic requires a comprehensive approach to prevent, treat and improve the living conditions. However, developing guidelines is required to better involve the various parties in the management of PLHIV for a better quality of life
\end{abstract}

Keywords: Oral Disease Management; People Living with HIV; Quality of Life.

\section{Introduction}

In recent decades, significant innovations have been made to manage HIV infection with the expansion of the therapeutic range. The introduction of highly active antiretroviral therapy [HAART] has contributed to the reduction of HIV infection-related morbidity and mortality. At the same time, the frequency and severity of HIV infection-related oral diseases have decreased significantly [1].

However, most people living with HIV [PLHIV] reside in developing countries and most of them do not have access to treatment. The HAART can predispose to human papillomavirus infection in the mouth and potentially increase the squamous oral carcinoma risk.

Despite efforts made in clinical care, most of the HIV-infected people worldwide will continue to develop oral pathologies [2]. However, many oral mucosal or dental conditions are an indicator of overall health. These oral manifestations sometimes reveal an overall pathology. Thus, dental professionals are often at the forefront, screening and preventing certain diseases. The accidental discovery of a lesion can engage professionals to initiate diagnostic steps (HIV screening) that often lead to general pathologies such as HIV. Indeed, detecting precancerous or cancerous oral lesions, or recurrent opportunistic lesions are among the first warning signs of an HIV infection. For example,

\footnotetext{
* Corresponding author: Mbathio Diop
} 
Kaposi's Sarcoma, which is an inaugural tumor of HIV infection, as well as oral hair leukoplakia, are set out in the Centers for Disease Control's guidelines [3]. Oral lesions associated with this disease are significant as they are useful markers of the disease progression that can affect the patient's quality of life [4].

Pandemic management requires a comprehensive approach to prevention, treatment and improvement of living conditions $[4,5,6]$. For this reason, this study had focused on dentists' role in the overall care of PLHIV.

\section{Material and methods}

\subsection{Documentary research}

The first step to carry out this study was to identify and collect the documentation. Thus, Pubmed, Medline, Pascal and Cochrane databases were consulted primarily. The choice of these databases was based on their contents' credibility. The "snowball" documentary research method which consisted of finding some strategic articles and searching for others through their references was used. Publications between 2000 and 2018 were used. Forty-Five articles out of the 152 consulted were selected.

Keywords used were people living with HIV + oral health promotion + quality of life. These words were combined with the Boolean operators "AND", "OR", "EXCEPT". Thus, abstracts of relevant articles were identified, including international conferences on HIV and oral health, their management and quality of life. The grey literature whose research was done on sites such as WHO, UNAIDS, Centers for Disease Control and Prevention, Ministry of Health and Solidarity of France was consulted too.

\subsection{Data analysis}

All electronically or bibliographically identified abstracts were analyzed. Where required, the full text was obtained for further information. The information obtained was gathered for use to confirm, or otherwise, the research hypotheses.

\section{Results}

\subsection{Early detection}

Oral health is an essential component of overall health. Thus, the oral cavity is not an isolated anatomical entity, but an integrated element into a system. As a result, dental surgeons' role goes beyond dental treatments and extractions, as they have a pivotal role in the early detection and prevention of certain diseases. Thus, dentists play a great part in early identification of signs and symptoms of HIV infection and its progression [6, 7].

Diagnosing the disease through the oral cavity has broader benefits than its local treatment because it enables early management of PLHIV. Clinical examination of the oral cavity is necessary, as oral lesions are indicators of the HIV infection progression. Indeed, there are effective treatments available; however, the people who should benefit from them, sometimes, are unaware of their HIV status. Thus, oral clinical examination allows early detection of HIV infection, which improves its prognosis and reduces its spread.

In Africa, besides the geographical and financial inaccessibility, there is a shortage of trained oral health professionals.

Thus, effectively assessing HIV infection-related risks during the clinical examination could increase their early detection. Several authors who share the same idea have demonstrated these results [5, 6, 7].

On the other hand, Alves et al. show that oral lesions used as seroconversion indicators are only limited to the candidiasis study. These results highlight the importance of using this oral candidiasis prevalence on early HIV detection and as a highly effective indicator of the disease's seroconversion. These data corroborate those in the literature in terms of appropriateness of oral candidiasis, which most often indicates severe immunosuppression [8]. Considering oral examination to detect HIV infection may be secondary in some developed countries since they can offer systematic screening, including serological tests that may be more clear and accurate.

However, the accidental discovery of HIV infection during dental office care is common in developing countries where the supply is lower than the demand for patients who are unaware of their health status. It is, without any doubt, a necessary step so that the pathology does not evolve towards a terminal stage to render its care intense. 
In short, lesions suggestive of candidiasis, Kaposi, recurrent infections, advanced disease, etc. would be serologically tested. While oral examination should not be a substitute for serology to detect the HIV progression, it has the benefit of providing guidance and early warning on the disease progression [1].

Promoting oral health actively involves the care of PLHIV.

\subsection{To communicate and promote oral health}

Oral health services aim to reduce the disease burden from the populations' daily living. As a result, dental services can, therefore, play a role in primarily preventing the HIV epidemic, particularly on its transmission method. They can also be involved in the early diagnosis and treatment of oral diseases related to the infection by promoting oral health. PLHIV still have difficulty accessing dental care, due to the volume of care provided compared with their needs and the acceptability of care. Social inequalities often complicate this inaccessibility to care [5].

Given these health facilities' inadequacy, dentists should emphasize the teaching to improve oral hygiene for reduced therapeutic needs.

Beyond its positive impact on oral health and the overall health throughout the individual's life, the fluoride is essential in bones and teeth mineralization. The proper use of topical or systemic fluoride has significantly reduced dental decay and its side effects. Because PLHIV susceptibility to dental caries is a given, especially in children, it would be wise to use fluoridation to prevent caries $[9,10]$.

Dental hygiene principles consist of:

- regular visits to the dentist (2 to 3 times a year);

- water fluoridation by WHO recommended standards of $1.5 \mathrm{mg} / \mathrm{L}$;

- toothpaste fluoridation (1,500ppm of fluoride for adults and $450 \mathrm{ppm}$ for children under the age of six);

- use of a soft toothbrush (to be changed every 3 months) or a toothbrush stick;

- brushing teeth (vertically from top to bottom) after meals for 2 minutes;

- a balanced diet while avoiding snacking on sweets (favoring fruit and vegetables, water and milk instead of fruit juices).

Indeed, nearly 100 national and international organizations have agreed on water fluoridation as an effective means to prevent dental caries, hence the necessity to include it in public health policies. Nutritionist professionals should regularly review and promote the use of systemic and topical fluorides, especially in children and adolescents living with HIV $[3,11]$.

In 2004, Hilton corroborated the same view on the importance of teaching good oral hygiene practices. The study on preventing recurrence of candidiasis shows a recurrence rate of $78 \%$ at six months among participants who received interventions compared to $88 \%$ for those in the control group. These results indicate that regular training by health professionals helps patients delay the recurrence of candidiasis by improving their oral hygiene. Among the PLHIVs, those with poor oral hygiene and a high sugar diet are most likely to be affected by these infection-related oral pathologies [12].

Besides promoting oral health, the dentist is involved in raising awareness and preventing HIV infection. Indeed, along with the counseling training, the oral health professional will be able to participate in the information, education and communication [IEC] campaign to eradicate HIV. This training also allows a rapid screening testing in remote areas before managing HIV-associated oral lesions.

\subsection{Office-based treatment of PLHIV}

Changes in medical therapy have improved the prognosis for infected HIV people. Nevertheless, it seems that the more there is resistance to treatment, the more progress towards immunodeficiency (AIDS stage) will occur. The current therapy has improved infected people's quality of life: however, it is not a cure for HIV infection. Therefore, the persistence of the number of HIV infection-related oral diseases requires the management of these lesions [13, 14].

This care is the result of good hygiene in dental offices, treatment of injuries and support for PLHIV to improve their quality of life. 


\subsubsection{Hygiene in dental practices}

Most often, the practitioner is unaware of the patient infectious status. The prevention of infection risk is a set of measures implemented to ensure the safety of patient's and healthcare professionals to prevent the risk of crossinfections and nosocomial infections transmission. Furthermore, dentists are particularly exposed to blood and biological products, as well. Their daily routine involves many invasive acts, including the use of very complex tools [15, 16].

Rutalaet al. have demonstrated in 2004 that these procedures' major risk is the potential infection by pathogens. The disinfection or sterilization of reusable medical tools has a breach of host barriers-related risk. The level of disinfection or sterilization depends on the intended use of the tool. For this reason, there are three categories of clinical tools:

- critical tools made up of surgical instruments, which are in contact with tissues (forceps, curettes, elevators, syndesmotomes);

- $\quad$ semi-critical tools, which include endoscopes and that are in contact with membranes and mucous membranes (precelle, mirror, turbine);

- non-critical tools such as their surface of which is not in direct contact with the skin.

It is essential to comply with basic hygiene measures and first among them the staff hygiene, aseptic and antiseptic.

\subsubsection{Staff hygiene}

Hygiene in the dental office is essentially based on knowledge and implementing preventive measures with regard to all patients. These standard precautions include wearing masks, gloves, glasses, lab coat and hand washing between patients, etc. They will help avoid blood and biological liquids-related contamination risk. Adhering to aseptic rules and preparing the patient help control the contamination $[15,16]$. Indeed, the self-assessment in the study by Fabiani et al. [17] shows the level of knowledge on infectious risks in the dental office to be good with $24 \%$ of practitioners. Although they have been vaccinated, most dentists (57\%) consider the Hepatitis B virus to be the main infectious agent the most feared. These professionals do not equally fear other diseases infection, such as respiratory infections and do not even mention the HIV infection. A chair reserved for surgery was considered by $90 \%$ of participants as an index of good hygiene and health education [18]. Despite the use of masks, disposable gloves, glasses and cups, few dentists change their lab coat or wash their hands between patients. Hydro alcoholics in dental surgery should be fully investigated to facilitate this protocol.

All of these rules must be followed in any place of care, by all practitioners and their assistants, and for any patient.

The quality of the environment, the preparation of the patient, the proper use of antiseptics and following rules of asepsis during any surgical act are essential to control the dental act-related infectious risk.

Certain things, such as staff hygiene, the maintenance of medical tools and the environment, assist with the asepsis of dental act $[18,16]$.

\subsubsection{Asepsis}

The effectiveness of the sterilization process depends directly on the proper completion and quality of previous steps (decontamination, cleaning, rinsing and drying). Cleaning must always precede high-level disinfection and sterilization.

Steam sterilization is the principle method from what is known at present. Thus, handling dental instruments can be deemed effective as long as they are sterilized in an autoclave (97\%) and periodically undergo sterilization effectiveness tests $(76 \%)[17]$.

To date, other sterilization methods have not been recommended for common practice in dental surgery and stomatology [18].

Indeed, sterilization requires to be held for 18 minutes at $134^{\circ} \mathrm{C}$ temperature.

Dry heat sterilization should be prohibited because the efficiency is random at the heart of the load and is inefficient on certain agents, such as viruses [44]. Yet, in Africa, practitioners have settled into it and still use the poupinel with certainty. Besides, it requires a cost of direct concerns to authorities of developing countries. 


\subsubsection{Antiseptic}

Using antiseptic in dental practices aims to reduce the microbial flora of the mouth, as well as the perioral cutaneous flora, which is likely to cause an infectious complication related to the care. Consequently, the activity is essentially bactericidal and fungicidal. Regarding veridical and sporicidal activities, they involve contact times and often concentrations of active constituents, well above those compatible with care practice $[7,16]$. The choice of an antiseptic will take into account its spectrum of antimicrobial activity and the contact time necessary for its effectiveness. In the dental sphere, its use on a mucosa restricts the use of certain products (such as highly concentrated alcohols). Many products contain combinations of synergistic molecules that increase product effectiveness. Knowledge of biological products, toxicological risks and preventive procedures improves the measures for professional practice.

\subsubsection{Preventing blood and body fluids exposure accidents}

Blood exposure accident [BEA] is any percutaneous exposure (by injection or cut) or contact with injured skin or mucous membranes (mouth, eyes) with blood or blood-contaminated body fluids.

Ergonomics, work method and planning enable both the assistant and the practitioner to prevent BEA. Cleaning and storing used tools and managing medical waste help to avoid BEA [15,16]. More than $64 \%$ of these accidents occur after treatment at the end of the dental procedure. When occupational exposure to HIV is suspected, it is recommended to apply post-exposure protocols. This further reduces the likelihood of HIV infection, stress and anxiety. It also allows the incident to be safely investigated and to protect those involved in the management of HIV infection [19].

However, implementing the ISO 9001:2008 standards related to hygiene and asepsis will be an effective tool to define an ethical framework and a common language and to determine service characteristics. It will also be a tool for progress and will enable oral health professionals to optimize the overall management of the dental practice and move towards quality $[20,21]$.

Once these prerequisites are met, the management of HIV infection-related oral conditions can be initiated. Thus, a dental practice in hospital structures facilitates access to dental care [22].

\subsection{Oral lesions treatment}

Broad access to HIV treatment has significantly reduced the prevalence of HIV infection-related oral lesions in Western countries. However, a potential increased prevalence of oral warts, a possible gap between CD4 cell levels and oral manifestations of HIV require the on-going attention of oral healthcare providers. Head, neck and oral cavity examination and carefully examining hypo salivation-related complications are essential components to a comprehensive oral health care program [23].

Oral and perioral lesions were common in HIV-positive adults and are treatable morbidity associated with this disease. Thus, a thorough oral cavity examination is important in the clinical assessment, management and follow-up of PLHIVs $[1,2,24]$.

The management of these patients requires overall care of all related conditions, such as periodontal diseases, fungal infections, viral infections, salivary glands diseases and neoplastic tumors.

\subsubsection{Periodontal diseases}

The spectrum of periodontal disease in HIV-infected people includes unusual forms of gingivitis, necrotizing diseases, advanced periodontitis and a multitude of adverse conditions to the oral mucosa. This gingivitis high resistance to regular treatment is a sign of chronic disease $[25,26]$. The treatment includes professional cleaning of the bacterial plaque, a necrotic debridement, mouthwash with antiseptic irrigation (chlorhexidine, povidone-iodine). In some cases, this treatment must be combined with systemic antibiotics. On the other hand, the Noma (necrotizing stomatitis) requires a much more complex treatment, which consists of wound debridement and sequestrectomy before the definitive reconstructive surgery. This treatment must also include antibiotic administration and nutritional support. Facial reconstruction is carried out using local and distant flaps. Bone reconstruction is sometimes necessary; however, it is often difficult to do a follow-up as these patients are out of sight six to 12 months after surgery [24].

To understand better HIV infection-related periodontal diseases, on-going research and an updated evidence-based design of treatment regimens are required [28]. 


\subsubsection{Fungal infections}

Human immunodeficiency virus-related oral candidiasis [OC] is common and often replicates in the form of the disease's initial signs. Untreated, these lesions will significantly contribute to the HIV infection-related morbidity. Treatment can be topical and systemic with antifungal drugs to treat oral candidiasis in HIV-infected patients.

Indeed, topical treatment with oral nystatin, clotrimazole or ketoconazole tablet is effective for oral candidiasis episodes. However, fluconazole is the referral treatment for recurrent oropharyngeal candidiasis. It has a better cure rate and is recommended for better relapse prevention. Tested for HIV infection-related candidiasis treatment, 50mg daily oral dose achieved a cure rate of $82 \%$. Similarly, fluconazole is effective for topical treatments of simple oropharyngeal candidiasis. However, patients relapse quicker than those treated with a systemic antifungal agent. The administration of Amphotericin B is effective for refractory candidiasis and is well tolerated [8, 29]. Nystatin appears to be less effective than clotrimazole and azoles for oropharyngeal candidiasis. Clotrimazole, as well as azoles, were found to be effective for clinical symptoms.

On the other hand, patients treated with fluconazole were more likely to remain disease-free during the follow-up period than those treated with other antifungal agents.

With evidence, Pienaar et al. [32] using the same procedure had demonstrated fluconazole efficiency. Comparing the clotrimazole, the itraconazole and the fluconazole in a therapeutic trial, the fluconazole proved to be the better mycological healer. In comparison with nystatin, fluconazole promotes clinical healing in adults. The two gentian violets having higher clinical healing than the nystatin, the fluconazole was compared with a placebo in a trial. In both cases, the fluconazole prevented clinical episodes. Fluconazole has the same prevention properties with on-going treatment $[7,30]$.

Anyways, the Candida organisms' potential resistance to drugs, as well as the cost of prophylaxis, could hinder this treatment.

Ketoconazole, fluconazole, itraconazole and clotrimazole improve treatment outcomes. The gentian violet and other less expensive antifungal drugs for OCs' treatment need more in-depth research.

However, they react favorably to antifungal drugs if their CD4/CD8 ratios had increased by 0.58 and 0.56 respectively. This report provides evidence of the levamisole efficiency in restoring T-cell immunity and helps to control oral candidiasis in patients who have undergone thymectomy.31

\subsubsection{Viral infections}

Leukoplakia

The oral leukoplakia is asymptomatic and has no potentiality malignant, it rarely requires complex treatment. Acyclovir and valacyclovir have been used for oral leukoplakia treatment [32].

Oral treatment with high doses of valacyclovir of nineteen cases of Oral hairy leukoplakia [OHL] and Epstein-Barr virus [EBV], which is an HIV infection-related replication virus, prevented the replication and production of EBV. The virus's clinical, histopathological and molecular responses to treatment were assessed in surgical biopsy samples obtained before, during and after treatment. In most cases, the OHL was cured and the BEV replication was stopped [33]. Nevertheless, in some cases, replication and production of BEV recur after the valacyclovir therapy is discontinued [34]. Unfortunately, resistance to acyclovir can prevent the clinical resolution of OHL [33]. In other cases where treatment has failed, EBV replication and production persisted, which could be due to its resistance to acyclovir.

Globally, the valacyclovir safe treatment of OHL and EBV replication provides new insights into mechanisms of the EBV persistence in the oral mucosa. Valacyclovir therapy completely abrogates EBV replication in vivo, resulting in the resolution of any existing OHL. Following the valacyclovir treatment, the EBV replication returned to the tongue normal epithelial cells [34].

\section{Kaposi's sarcoma}

Local therapy may be effective in the early stage of the tumor, but systemic therapy is necessary for diffused Kaposi's sarcoma [KS]. Indeed, the treatment of KS and HIV-related lymphedema focuses on the treatment of HIV, the KS and the lymphedema. HAART combined with cytotoxic chemotherapy is the management of HIV-associated KS referral 
treatment. This HAART therapy is useful in the management of HIV-related KSs, as it reduces the HIV viral load and increases CD4 percentage $[2,10]$. These two indicators contribute indirectly to Kaposi's sarcoma pathogenesis. Laser therapy, by surgical removal and cytotoxic treatment with periwinkle alkaloids (vinblastine, vincristine and vinorelbine) and bleomycin is effective. However, the Food and Drug Administration [FDA] has currently approved only five agents for the treatment of Kaposi's sarcoma, which are the topical treatment of alitretinoin gel, systemic treatment of liposomal daunorubicin, aldoxorubicin, paclitaxel and interferon-alpha [35].

Moreover, Kaposi's sarcoma-associated with HIV infection in combination with facial edema, in the absence of antiretroviral treatment appears to be of poor prognosis $[3,36]$.

\subsubsection{Damage to salivary glands}

Most pediatric patients with benign cystic lymphoid parotid gland disease associated with HIV infection can be treated by observation and with antiretroviral drugs. Sclerotherapy may offer a reasonable option while radiotherapy and surgery should only be done on the most complicated cases [37].

\section{Xerostomia}

The treatment of xerostomia has been very successful with broader topical and systemic ranges. A range of systemic therapy has been advocated for the management of xerostomia. Early intervention for dry mouth problems helps prevent the harmful consequences of this disease in affected people. Clinicians must be prepared to provide diagnosis and treatment to protect patients' oral and pharyngeal health $[3,37]$. This therapy principle is to improve the lubrication and hydration of oral tissues for good oral health.

Traditionally, the treatment of dry mouth focused on palliative measures to salivary substitutes. However, they have a short effect. The combination of mouthwash, toothpaste and chewing gum improves xerostomia symptoms. Indeed, sugarless gum or sugar-free candy can increase saliva secretion, but they can have some side effects and affect the patients' compliance with treatment. Lubricants in the form of gels, mouthwash and tablets have been used and they yielded varying results that relieve xerostomia symptoms.

Likewise, the mucin spray was found to be useful against xerostomia in those who had undergone irradiation. Recently, systemic pilocarpine has been found to be effective in managing radiation-induced xerostomia. Its use can significantly improve strategic management by stimulating secretion by salivary glands. Other methods of stimulating salivation, such as chewing gum, can improve salivary secretion through salivary glands. However, its disadvantage is that these actions are likely to be transient and it is prohibited in patients with full prostheses. Anticholinergic agents are promising and are appropriate for the treatment of radiation-related xerostomia.38

Hypertrophy of the salivary glands

The treatment of salivary gland hypertrophy in HIV disease remains nonspecific. However, clinical photographs and computerized tomography support the regression of the parotid gland following an ARV treatment. This success reflects a decrease in viral load and immune restoration. Superficial parotidectomy has been advocated for the reduction of HIV infection-related parotid volume, but its application may be constrained due to the related morbidity. Aspiration of cystic lesions may be useful but transient, and multiple cysts hinder the tetracycline and doxycycline injections success. Reconstructive surgery is possible in patients with low CD4/CD8 ratios due to HIV infection [39].

\subsubsection{Neoplastic tumor}

Soft tissue masses characterize oral manifestations of Non-Hodgkin's Lymphoma [NHL], with or without ulceration and tissue necrosis, usually involving the gum, the palate and the alveolar mucosa. The HIV-related NHL optimal treatment is uncertain. However, its management by oral route mainly involves systemic treatment [14].

The introduction of HAART has radically changed the disease sphere. The protease inhibitor and antiretroviral therapy available since late 1995 have reduced deaths from AIDS-related diseases by $75 \%$.

Although KS is decreasing, the NHL is more complicated. Even though the central nervous system lymphoma incidence is reduced, the stable number of potential patients with this systemic pathology is relative. The multi-therapy has not significantly reduced the AIDS-related NHL and is very challenging in the field of AIDS oncology. The focus is now on healing while continuing to closely monitor the particular vulnerability of HIV infected people [3]. 
In studies of Africa, the HIV-related NHL risk is about ten times lower than in developed countries, which is certainly due to the early death of PLHIV in developing countries. This may explain the relative absence of HIV-related lymphomas.

The treatment of HIV infection-related pathologies in children is generally similar to that of adults, but there are some differences, particularly about the management of cavities and possible tooth eruption.

Children infected with HIV may be more vulnerable to tooth decay. Therefore, their management must include the treatment of dental caries. In addition, previous periodontal disease could accelerate tooth eruption, but the exact cause of delayed tooth eruption is unknown. These children have generally poor health status, especially when associated with malnutrition, which can be a significant ulcerative gingivitis risk factor [40].

Therefore, the periodontal treatment plan will range from oral swabbing with mouthwash to complex surgery, depending on the severity of the lesions to avoid potentially fatal complications. Furthermore, chlorhexidine $(0.12 \%$ CHX) disinfectant can be a promising agent for the treatment and prevention of oral candidiasis in HIV-infected children [41].

However, confirmed HIV infected children should receive appropriate dental care, curative as well as preventive treatment [40].

At the same time, parents must be made aware and need to be informed on children's right oral health behavior, such as brushing teeth with fluoridated toothpaste and a balanced diet.

We cannot ignore the level of PLHIV's satisfaction in health facilities. Assessing HIV patients' access and satisfaction with dental services shows that, overall, respondents were satisfied with the dental care they had received. Most patients (58\%) were new to the practice and were more likely to report that a dentist had not seen them for more than 12 months. When asked about their expectations, they would like more access to dental care, the assessment of their needs, and to be made comfortable [22].

Therefore, it is necessary to consider these parameters in treatment guidelines for PLHIV. The research community

\subsection{Support in terms of quality of life}

HIV infection-related oral lesions cause severe pain, high morbidity and can compromise the aesthetics. They can also compromise adequate nutrition, good oral hygiene and further deteriorate the patient's health to accelerate the progression of the disease. However, early detection and diagnosis of these oral lesions by the dentist offers the patient quick and appropriate treatment and advice [1].

Interventions intended to prevent and treat HIV-related Candida oral lesions are a key component in maintaining HIV infected patients' quality of life [4].

Furthermore, the study by Yengopal et al. [42] on the impact of oral lesions on health insurance showed that HIV patients have a compromised quality of life. Indeed, this deterioration involves functional limitation, physical pain and psychological discomfort. Thus, more than $50 \%$ of patients have reported being uncomfortable in eating certain food, nearly $30 \%$ of patients have reported that their general health had worsened due to oral problems, and for $26.7 \%$ of patients, life was generally less satisfactory. The most common oral cavity symptoms reported are dry mouth (34.4\%), difficulty eating $(27.9 \%)$ and pain $(27.3 \%)[24]$.

Thus, nutrition and oral health interact in practice. Education and research in both fields (dietetics and dentistry) should be a priority. Due to the extent and impact of these conditions on nutritional status (HIV), a dental intervention, in conjunction with nutrition management, is essential to the care. HIV patients are at risk of oral diseases that lead to difficult nutritional support and systemic consequences. Fungal oropharyngeal infections can cause a burning sensation, oral pain and dysphagia. Viral oral diseases, such as simplex herpes and cytomegalovirus can cause chronic pain (painful ulcers). These problems, as well as stomatitis and periodontitis associated with pain, compromise the appetite and can lead to nutrition reduction. The same is true for oral cancers, such as the oral malignant tumor in HIV-positive patients [9].

Therefore, a good nutritional intake is necessary to consolidate the oral mucosa, which ensures the natural defense system of the oral cavity. 
Nutrition is an integral component of oral health. Thus, dietitians and dental professionals need to collaborate to promote oral health and prevent diseases [42]. Oral manifestations, associated with systemic diseases, confirm their impact on the nutritional status and the correlation between functional ability to eat and diet. Similarly, nutrition and diet can affect the development and integrity of the oral cavity and the progression of oral diseases. Furthermore, oral health professionals and nutritionists should provide the patient with basic education and guidance in comprehensive care.

\section{Conclusion}

A failed immune system enables infections thus rendering PLHIVs vulnerable. They require intense care along with easy access to health services where financial resources are limited. PLHIV's health status indicates high morbidity and mortality and, above all, a high prevalence of oral lesions that reflect compromised overall health. Without proper functioning of their oral cavity, PLHIVs are unable to eat properly to restore their body balance, which will negatively affect their quality of life. Besides, just medical therapy and nutritional support are not enough for the care of PLHIV who need psychosocial support to cope with their disease. On the psychological level, support should be provided through counseling, referrals to appropriate referral structures including access to antiretroviral drugs and their integration into PLHIV associations. Socially, these PLHIV are often stigmatized and discriminated against, so they need support to deal with this chronic disease many related problems. This support should be provided through community mobilization and awareness raising on the integration of PLHIV by involving the media. This will facilitate their acceptance by the population. However, the application of the ISO 9001:2008 standards related to hygiene and asepsis will be an effective tool and a vector of progress that will enable oral health professionals to optimize the overall management of the dental practice to avoid nosocomial infections.

\section{Compliance with ethical standards}

\section{Disclosure of conflict of interest}

The authors declare they have no conflict of interest related to this manuscript.

\section{References}

[1] Moodley A and Wood NH. (2015). HIV-Associated Oral Lesions in HIV-Seropositive Patients at an HIV-Treatment Clinic in South Africa. J AIDS Clin Res, 6(2), 422-32.

[2] Ross JM, Ying R, Connie L, Celum CL, Baeten JM, Thomas KK et al. (2018). Modeling HIV disease progression and transmission at population-level: The potential impact of modifying disease progression in HIV treatment programs. Epidemics, 23, 34-41.

[3] Mathur S, Jani M, Thakkar P and Shah A. (2014). Oral Manifestations of HIV Infection. Int J Dent Med Res, 1(4), $139-42$.

[4] Jha R, Kaur T and Sharma A. (2014). Oral Manifestations of HIV-AIDS: A Diagnostic and Management Dilemma. J Res Med Deb Sci, 2(1), 96-101.

[5] Awofala AA and Ogundele OE. (2018). HIV epidemiology in Nigeria. Saudi Journal of Biological Sciences, 25, 697703.

[6] Ba B, Konate I, Goïta D, Armel MG, Coulibaly A, Keita K, et al. (2017). Affections bucco-dentaires associées à l'infection à VIH dans le service de maladies infectieuses du CHU de Point-G, Bamako. Med Buccale Chir Buccale, 23, 5-11.

[7] Navazesh M. (2014). Thirty Years of HIV/AIDS and Related Oral Manifestations and Management. JDH, 88(3), 153-5.

[8] Alves TP, Simões AC, Soares RM, Moreno DS, Portela MB and Castro GF. (2014). Salivary lactoferrin in HIVinfected children: correlation with Candida albicans carriage, oral manifestations, HIV infection and its antifungal activity. Arch Oral Biol, 59(8), 775-82.

[9] Decker R, Touger and Mobley CC. (2007). American Dietetic Association. Position of the American Dietetic Association: the impact of fluoride on health: Oral health and nutrition. J Am Diet Assoc, 107(8), 1418-28. 
[10] Aleixo RQ, Scherma AP, Guimarães G, Cortelli JR and Cortelli SC. (2010). DMFT index and oral mucosal lesions associated with HIV infection: cross sectional study in Porto Velho, Amazonian Region - Brazil. Braz J Infect Dis, 14(5), 449-56.

[11] Portela MB, Chagas MSD, Cerqueira DF, De Souza IPR, Souto-Padrón T, Soares RMDA, et al. (2012). Differential collagenolytic activity of Candida albicans isolated from oral mucosa and dentinal carious lesions of HIV-infected children. Oral Surg Oral Med Oral Pathol Oral Radiol, 113, 378-83.

[12] Hilton JF, MacPhail LA, Pascasio L, Sroussi HY, Cheikh B, LaBao ME, et al. (2004). Self-care intervention to reduce oral candidiasis recurrences in HIV-seropositive persons: a pilot study. Community Dent Oral Epidemiol, 32(3), 190-200.

[13] Agwu E, Ihongbe JC, Ezeonwumelu JO and Lodhi MM. (2015). Baseline burden and antimicrobial susceptibility of pathogenic bacteria recovered from oral lesions of patients with HIV/AIDS in South-Western Uganda. Oral Science International, 12, 59-66.

[14] Lazare S, Devoize L, Jacomet C, Cormerais L, Orliaguet T and Baudet-Pommel M. (2005). Affections buccales classantes dans l'infection à VIH: analyse rétrospective de 62 patients sur 4 ans. Med Buccale Chir Buccale, 11, 195-204.

[15] Alapatt JG, Varghese NM, Joy PT, Saheer KM and Correya BA. (2016). Infection Control In Dental Office: A Review. J Dent Med Sci, 15(2), 10-5.

[16] Ministère de la santé et des solidarités de la France. (2006). Guide de prévention des infections liées aux soins en Chirurgie Dentaire et en stomatologie. Guide d'asepsie en France, (2), 27-31.

[17] Fabiani L, Mosca G and Giuliani AR. (2006). Hygiene in dental practices. Eur J Paediatr Dent, 7(2), 93-7.

[18] Burgess J. (2016). HIV and Dental Treatment JSM Dentistry, 4(2), 1062-7.

[19] Scully C and Greenspan JS. (2006). Human immunodeficiency virus (HIV) transmission in dentistry de. Res Dent J, 85(9), 794-800.

[20] Bourgeois D and Comte B. (2008). Hygiène et asepsie à l'usage des cabinets dentaires. Guide de lecture ISO 9001.

[21] Bissagnéné E, Dariosecq JM, Inwoley A, Taburet AM and Traoré HA. (2009). Mémento thérapeutique du VIH / SIDA en Afrique. Paris: Wolters Kluwer France Deuxième édition, 167.

[22] Lemay CA, Kretsedemas M and Graves JR. (2010). Satisfaction with dental case management among people living with HIV/AIDS. J Community Health, 35(1), 43-52.

[23] Sroussi HY and Epstein JB. (2007). Changes in the pattern of oral lesions associated with HIV infection: implications for dentists Can Assoc J Dent, 73(10), 949-52.

[24] Guteta S, Feleke Y, Fekade D, Neway M and Diro E. (2008). Prevalence of oral and perioral manifestations in HIV positive adults at Tikur Anbessa Teaching Hospital Addis Ababa, Ethiopia Ethiop Med J, 46(4), 349-57.

[25] Toljić B, Trbovich AM, Petrović SM, Kannosh IY, Dragović G, Jevtović D, et al. (2018). Ageing with HIV - a periodontal perspective. New Microbiologica, 41(1), 61-6.

[26] Oliscovicz NF, Pomarico L, Castrob GFBDA and Souza IPR. (2015). Effect of highly active antiretroviral therapy use on oral manifestations in pediatric patients infected with HIV. Ind J Dent Res, 26(2), 200-4.

[27] Chidzonga MM and Mahomva L. (2008). Recurrent noma (stomatites) (cancrumoris) in human immunodeficiency virus infection and acquired immunodeficiency syndrome (HIV and AIDS): report of a case. J Surg Oral Maxillo-Faciale, 66(3), 475-85.

[28] Arrive E, Kouakoussui A, Eboumou E, Akatia F, Rouet F and Msellati P. (2010). Carie dentaire, gingivite et VIH pédiatrique en Côte d'Ivoire. Actualités Odonto-Stomatologiques, 252, 375-83.

[29] Albougy HA and Naidoo S. (2002). A systematic review of the management of oral candidiasis associated with HIV/AIDS. SADJ, 57(11), 457-66.

[30] Pienaar ED, Young T and Holmes H. (2006). Interventions for the prevention and management of oropharyngeal Pública candidiasis associated with HIV infection in adults and children. Cochrane Database Syst Rev, 19, 3, CD003940.

[31] Lai WH, Lu SY and Eng HL. (2002). Levamisole aids in treatment of refractory oral candidiasis in two patients with thymoma associated with myasthenia gravis: report of two cases. Chang Gung Med J, 25(9), 606-11. 
[32] Petruzzi MNMR, Cherubini K, Salum FG and De Figueiredo MAZ. (2013). Risk factors of HIV-related oral lesions in adults. Rev Saúde, 47(1), 52-9.

[33] Walling DM, Flaitz CM, Nichols CM, Hudnall SD and Adler-Storthz K. (2001). Persistent productive Epstein-Barr virus replication in normal epithelial cells in vivo. J Infect Dis, 184(12), 1499-507.

[34] Robinson PG. (2006). Implications of HIV disease for oral health services. Adv Dent Res, 19(1), 73-9.

[35] Dezube BJ. (2002). Management of AIDS-related Kaposi's sarcoma: advances in target discovery and treatment. Expert Rev Anticancer Il, 2, 193-200.

[36] Feller L, Masipa J, Wood N, Raubenheimer E and Lemmer J. (2008). The prognostic significance of facial lymphoedemain HIV-seropositive subjects with Kaposi sarcoma. AIDS Res Ther, 5, 2.

[37] Dave SP, Pernas FG and Roy S. (2007). The benign lymphoepithelial cyst and a classification system for lymphocytic parotid gland enlargement in the pediatric HIV population. Laryngoscope, 117(1), 106-13.

[38] Baccaglini L, Atkinson JC, Patton LL, Glick M, Ficarra G and Peterson DE. (2007). Management of oral lesions in HIV-positive patients. Oral Surg Oral Med Oral Pathol Oral Radiol Endo, 103(50), e1-23.

[39] Tindyebwa D, Kayita J, Musoke P, Eley B, Nduati R, Coovadia H, et al. (2006). Manuel sur le SIDA pédiatrique en Afrique. Réseau Africain pour les soins aux enfants affectés par le SIDA, 286.

[40] Khongkunthian P0, Grote M, Isaratanan W, Piyaworawong S and Reichart PA. (2001). Oral manifestations in 45 HIV-positive children from Northern Thailand. J Pathol Oral Med, 30(9), 549-52.

[41] Eldridge K and Gallagher JE. (2000). Dental caries prevalence and dental health behaviour in HIV infected children. J Dent Paed Int, 10(1), 19-26.

[42] OMS. (2006). Plan d'action pour la promotion de la santé bucco-dentaire et pour la prévention intégrée des affections. Conseil Excécutif.

[43] Rutala WA and Weber DJ. (2004). Disinfection and sterilization in health care facilities: what clinicians need to know. Clin Infect Dis, 39(5), 702-948.

[44] Yengopal V and Naidoo S. (2008). Do oral lesions associated with HIV affect quality of life? Oral Surg Oral Med Oral Pathol Oral Radiol Endo, 106(1), 66-73.

[45] Younai FS, M Marcus, Freed JR, Coulter ID, W Cunningham, C-Martirosian Der, et al. (2001). Self-reported oral dryness and HIV disease in a national sample of patients receiving medical care. Oral Surg Oral Med Oral Pathol Oral Radiol Endo, 92, 629-36.

\section{How to cite this article}

Diop M, Diatta M, Kanouté Aida, Dieng SD, Ndiaye M, Bourgeois D, Lo CMM and Faye D. (2020). People living with HIV and oral health professionals: What relationship? World Journal of Advanced Research and Reviews, 7(2), 45-55. 